\title{
Periodic Motion and Transition of a Vibro-Impact System with Multilevel Elastic Constraints
}

\author{
Jie Ding, Chao Wang, and Wangcai Ding \\ School of Mechatronic Engineering, Lanzhou Jiaotong University, Lanzhou 730070, China \\ Correspondence should be addressed to Wangcai Ding; dingdd@163.com
}

Received 14 December 2020; Revised 14 January 2021; Accepted 22 January 2021; Published 9 February 2021

Academic Editor: Viktor Avrutin

Copyright ( 2021 Jie Ding et al. This is an open access article distributed under the Creative Commons Attribution License, which permits unrestricted use, distribution, and reproduction in any medium, provided the original work is properly cited.

\begin{abstract}
In this paper, a single-degree-of-freedom vibroimpact system with multilevel elastic constraints is taken as the research object. By constructing the Poincaré map of the system and calculating the Lyapunov exponent spectrum of the system, the stability of the system is determined. Using the multiparameter collaborative numerical simulation method, the parameter domains of various periodic motions are determined, and the diversity and transition characteristics of periodic motions are revealed. At the same time, combined with the cell mapping method, the coexistence of attractors induced due to grazing bifurcation, saddle-node bifurcation, and boundary crisis is studied. Finally, the influence of system parameters on periodic motion distribution is analyzed, which provides a scientific basis for system parameter optimization.
\end{abstract}

\section{Introduction}

The vibroimpact system with clearance, as a typical nonsmooth dynamic system, is widely used in engineering practice, such as vibrating sand shaker [1], vibration isolation system with actuators [2], impact damping device [3], railway train wheel-rail vibration [4], and vibroimpact capsule system [5]. This kind of system exhibits rich and complex nonsmooth dynamic behavior due to the sudden change of stiffness caused by clearance and elastic constraints.

In the past few decades, many scholars have conducted extensive research on the segmented smooth impact vibration system with clearance, using analytical and numerical methods. Shaw and Holmes [6] first studied the piecewise linear oscillator under simple harmonic excitation with the view of modern dynamic system, analyzed the partial bifurcation of periodic motion by the central manifold theorem, and discussed the chaotic motion by the homoclinic phase intercept condition. Natsiavas $[7,8]$ proposed a joint method with a relatively small computational workload and studied the resonance response of symmetric and asymmetric piecewise linear oscillators excited by simple harmonics. Choi and Noah [9] introduced the fast Fourier transform in the harmonic balance method to calculate the harmonic coefficients of each order of the nonlinear restoring force and then studied the multiple resonance responses of the asymmetric elastic constrained oscillator. Ketema [10] applied the Melnikov method to the determination of the existence of the homoclinic point of the Poincaré mapping of the piecewise linear oscillator system. $\mathrm{Hu}$ [11] used a combination of segmented analytical solutions and shooting methods to improve the accuracy and efficiency of calculating the periodic motion of the elastic restraint system. Luo [12] used a nonsmooth analysis method to study various unstable and stable periodic motion patterns of the piecewise linear periodic excitation system.

The instability, caused by the grazing impacts in the vibration shock system, is a hot spot of current research. As a pioneer in this field, Nordmark [13] studied the nonperiodic motion caused by the grazing impacts in the piecewise linear system. Chin et al. [14] analyzed a single-degree-of-freedom vibration system and proposed several different types of edge rubbing and bifurcation that caused changes in system dynamics. Chillingworth [15] studied the dynamic behavior of a single-degree-of-freedom shock oscillator near the edge rubbing point, including nondegenerate (secondary rubbing) and minimal degradation (three rubbing) phenomena. 
Humphries et al. [16] introduced a discontinuous geometry topology method to explain the cause of saddle-knot bifurcation near the edge rubbing bifurcation. Kryzhevich and Wiercigroch [17] studied the existence of nonhyperbolic homoclinic points of the periodic solutions of vibration shock systems and introduced a new type of robust chaotic dynamics for strongly nonlinear systems. Pavlovskaia et al. [18] used a large number of experiments to study the impact vibrator with unilateral elastic restraint and revealed the influence of different excitation frequencies near the edge rubbing point on the bifurcation. The result showed that there was attractors coexistence phenomenon when there was discontinuous transition among the two orbits via boundary crisis. Jiang et al. [19] used the path tracking method to study the dynamic behavior of the grazing bifurcation of the elastic and rigid impact vibrators. Under the constraints of the unilateral elastic impact vibrator, the smooth bifurcation (period-doubling, saddle-node) point was close to nonsmooth (grazing) bifurcation points, and there was no smooth bifurcation near the nonsmooth bifurcation point under the constraints of the unilateral rigid impact oscillator.

The cell mapping method, created by the American mathematician Hsu [20] in the 1980s, is an effective tool for studying the global characteristics of nonsmooth dynamic systems. The advantage of this method is that it can efficiently and dynamically analyze the attractors, attraction domain, and chaos of the system. The behavior more comprehensively reveals the mechanism of the periodic movement of the system. Based on the idea of the cell mapping method, many scholars have proposed simple cell mapping [21], interpolated cell mapping [22], graph cell mapping [23], and other improved cell mapping methods and obtained a lot of results in the quantitative and qualitative analysis of the global dynamic characteristics of nonsmooth dynamic systems. Kong et al. [24] used the generalized cell mapping method to study the global dynamics of piecewise linear systems excited by a Gaussian white noise. Li et al. [25, 26] analyzed the diversity and evolution of basic periodic shock motions by combining the cell mapping method on the simple harmonic vibration dynamic model with gaps and studied the distribution of attractors and attractive domains. Chong et al. [27] studied in detail the influence of different parameters on system dynamics by combining the path following method and the cell mapping method for the harmonically excited linear oscillator with gaps.

At present, most actual mechanical systems with clearances are multiparameter and multiconstraint systems, and the coordinated changes of multiple parameters show the dynamic behavior of the system more comprehensively. However, the existing research is mostly limited to the case of a single parameter. This paper not only considers multiple constraint factors but also conducts numerical simulation under the coordinated change of multiple parameters. At the same time, combined with the cell mapping method, the dynamic characteristics of a class of impact vibration systems with multilevel elastic constraints are studied. The periodic motion distribution and the transition process of periodic motion through various nonsmooth bifurcations are analyzed. The structure of this paper is as follows. In the second section, the physical model and motion equations of the collision system with two elastic constraints are established. In Section 3, the Poincaré map and Lyapunov exponent of the system are derived, which can accurately determine the periodic motion and chaotic motion in the parameter plane. In Section 4, the distribution area of periodic motion and the transition law between adjacent periodic motions are studied through a multiparameter collaborative simulation, and the coexistence of periodic motion in the transition process of periodic motion is studied with the method of cell mapping. Section 5 studies the influence of system parameters on the distribution of periodic motion. Section 6 gives a summary of this article.

\section{Mathematical Modelling}

The physical model of a single-degree-of-freedom vibroimpact system with multilevel elastic constraints is shown in Figure 1. A vibrator with a mass $M$ is connected by a linear spring with a stiffness $K$ and a linear damper with a damping coefficient $C$ and moves along the horizontal direction under the action of harmonic exciting force $F_{0} \sin (\Omega T+\tau)$. The coordinate system is established by taking the static equilibrium position of the system as the origin of the spatial coordinates, the displacement of the vibrator is $X$, and the elastic constraints with stiffnesses $K_{1}$ and $K_{2}$ are fixed at positions $B_{1}$ and $B_{1}+B_{2}$ to the right of the vibrator. Under the action of the harmonic exciting force, when the displacement of the vibrator is small, the system is a single-degree-of-freedom linear vibration system; when the displacement of the vibrator is greater than $B_{1}$ or $B_{1}+B_{2}$, the vibrator will interact with one or two elastic constraints on the right side. The system will exhibit strong nonlinearity and nonsmooth characteristics due to the existence of clearances and the occurrence of collisions.

The mass $M$ is subjected by the spring force, damping force, and external exciting force at the same time. According to Newton's theorem, the differential equations of system motion can be expressed as

$$
\begin{gathered}
\left\{M \ddot{X}+C \dot{X}+K X=F_{0} \sin (\Omega T+\tau), X \leq B_{1},\right. \\
M \ddot{X}+C \dot{X}+K X+K_{1}\left(X-B_{1}\right)=F_{0} \sin (\Omega T+\tau), B_{1}<X<B_{2}, \\
M \ddot{X}+C \dot{X}+K X+K_{1}\left(X-B_{1}\right)+K_{2}\left(X-B_{1}-B_{2}\right)=F_{0} \sin (\Omega T+\tau), X \geq B_{1}+B_{2} .
\end{gathered}
$$




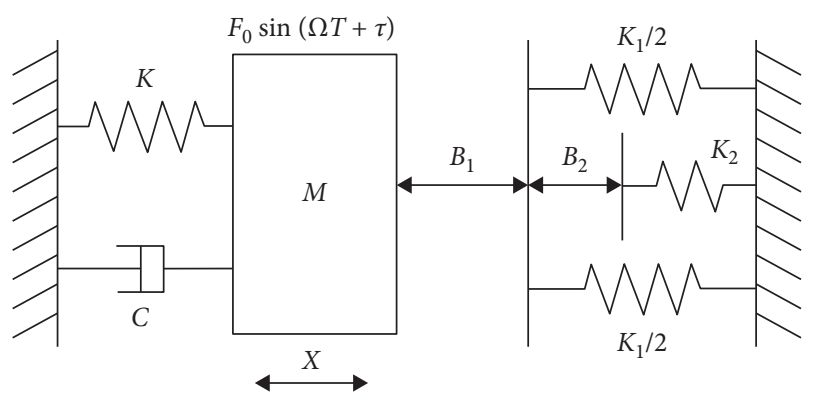

Figure 1: Physical model of a single-degree-of-freedom vibroimpact system with multilevel elastic constraints. mechanism of the mechanical model and eliminate the influence of dimensions, it is necessary to perform nondimensional processing on the differential equation of motion and introduce the following nondimensional quantities: $x=\left(X K / F_{0}\right), \quad b_{1}=\left(B_{1} K / F_{0}\right), \quad b_{2}=\left(B_{2} K / F_{0}\right), \quad \zeta=(C / 2$ $\sqrt{M K}), \quad \omega=\Omega \sqrt{M / K}, \quad t=\mathrm{T} \sqrt{K / M}, \quad \mu_{k 1}=\left(K_{1} / K\right)$, and $\mu_{k 2}=\left(K_{2} / K\right)$.

The nondimensional differential equations of the system motion are

Due to the different dimensions of these physical quantities, in order to thoroughly study the motion

$$
\begin{aligned}
\{\ddot{x}+2 \zeta \dot{x}+x & =\sin (\omega t+\tau), x \leq b_{1}, \\
\ddot{x}+2 \zeta \dot{x}+\left(1+\mu_{k 1}\right) & =\sin (\omega t+\tau)+\mu_{k 1} b_{1}, b_{1}<x<b_{1}+b_{2}, \\
\ddot{x}+2 \zeta \dot{x}+\left(1+\mu_{k 1}+\mu_{k 2}\right) & =\sin (\omega t+\tau)+\mu_{k 1} b_{1}+\mu_{k 2}\left(b_{1}+b_{2}\right), x \geq b_{1}+b_{2} .
\end{aligned}
$$

The general solutions of the set of differential equations

(2) are

$$
\left\{\begin{aligned}
x(t)= & e^{-\eta_{1}\left(t-t_{0}\right)}\left(a_{1} \cos \omega_{d 1}\left(t-t_{0}\right)+c_{1} \sin \omega_{d 1}\left(t-t_{0}\right)\right) \\
& +A_{1} \sin \left(\omega t+\tau_{0}\right)+C_{1} \cos \left(\omega t+\tau_{0}\right), \quad x \leq b, \\
x(t)= & e^{-\eta_{2}\left(t-t_{1}\right)}\left(a_{2} \cos \omega_{d 2}\left(t-t_{1}\right)+c_{2} \sin \omega_{d 2}\left(t-t_{1}\right)\right) \\
& +A_{2} \sin \left(\omega t+\tau_{0}\right)+C_{2} \cos \left(\omega t+\tau_{0}\right)+\frac{\mu_{k_{1}} b_{1}}{\left(1+\mu_{k 1}\right)}, \quad b_{1}<x<b_{1}+b_{2}, \\
x(t)= & -e^{-\eta_{3}\left(t-t_{2}\right)}\left(a_{3} \cos \omega_{d 3}\left(t-t_{2}\right)+c_{3} \sin \omega_{d 3}\left(t-t_{2}\right)\right) \\
& +A_{3} \sin \left(\omega t+\tau_{0}\right)+C_{3} \cos \left(\omega t+\tau_{0}\right)+\frac{\left(\mu_{k_{1}} b_{1}+\mu_{k_{2}}\left(b_{1}+b_{2}\right)\right)}{\left(1+\mu_{k 1}+\mu_{k 2}\right)}, \quad x \geq b_{1}+b_{2},
\end{aligned}\right.
$$

where $\omega_{n 1}^{2}=1, \quad \omega_{n 2}^{2}=1+\mu_{k}, \quad \omega_{n 3}^{2}=1+\mu_{k 1}+\mu_{k 2}, \quad \eta_{1}=\zeta$, $\eta_{2}=\zeta, \quad \eta_{3}=\zeta, \quad \omega_{d 1}=\sqrt{\omega_{n 1}^{2}-\eta_{1}^{2}}, \quad \omega_{d 2}=\sqrt{\omega_{n 2}^{2}-\eta_{2}^{2}}, \quad$ and $\omega_{d 3}=\sqrt{\omega_{n 3}^{2}-\eta_{3}^{2}} ; a_{1}, a_{2}, a_{3}, c_{1}, c_{2}$, and $c_{3}$ are the integral constants, respectively, which are determined by the initial conditions of the vibration system; $A_{1}, A_{2}, A_{3}, C_{1}$, $C_{2}$, and $C_{3}$ are the amplitude constants, respectively, which have 


$$
\begin{aligned}
& A_{1}=\frac{\left(1-\omega^{2}\right)}{\left(\left(1-\omega^{2}\right)^{2}+(2 \xi \omega)^{2}\right)}, \\
& C_{1}=\frac{-2 \xi \omega}{\left(\left(1-\omega^{2}\right)^{2}+(2 \xi \omega)^{2}\right)}, \\
& A_{2}=\frac{\left(\left(1+\mu_{k}\right)-\omega^{2}\right)}{\left(\left(\left(1+\mu_{k}\right)-\omega^{2}\right)^{2}+(2 \xi \omega)^{2}\right)}, \\
& C_{2}=\frac{-2 \xi \omega}{\left(\left(\left(1+\mu_{k}\right)-\omega^{2}\right)^{2}+(2 \xi \omega)^{2}\right)}, \\
& A_{3}=\frac{\left(\left(1+\mu_{k 1}+\mu_{k 2}\right)-\omega^{2}\right)}{\left(\left(\left(1+\mu_{k 1}+\mu_{k 2}\right)-\omega^{2}\right)^{2}+(2 \xi \omega)^{2}\right)} \\
& C_{3}=\frac{-2 \xi \omega}{\left(\left(\left(1+\mu_{k 1}+\mu_{k 2}\right)-\omega^{2}\right)^{2}+(2 \xi \omega)^{2}\right)}
\end{aligned}
$$

\section{Poincaré Mapping of the System}

In order to study the stability, diversity, existence area, and local bifurcation of the periodic motion of the system, the Poincaré mapping of the system is established.

Select the mapping section $\Sigma_{T}=\left\{(x, \dot{x}, t) \in R^{2} \times S, x=\right.$ $\left.x_{-}, \bmod (t=2 \pi / \omega)\right\}$ to count the number of cycles of periodic motion of the system; use collision surfaces $\Sigma_{p 1}=$ $\left\{(x, \dot{x}, \theta) \in R^{2} \times S, x=b_{1}, \dot{x}>0\right\}$ and $\Sigma_{p 2}=\left\{(x, \dot{x}, \theta) \in R^{2} \times S, x=b_{1}+b_{2}, \dot{x}>0\right\}$ to count the number of collisions between the oscillator and the constrained surface.

The Poincaré mapping diagram of the system is shown in Figure 2. The collision surface $\Sigma_{p 1}$ is used to establish the Poincaré mapping of the system: $P: \Sigma_{p 1} \longrightarrow \Sigma_{p 1}$; the eigenvalues of the linearization matrix of the Poincare mapping at the fixed points are used to study the stability of the system's periodic motions. According to the contact process, Poincaré mapping is divided into five stages: $P_{1}$ : the vibrator does not contact with the linear springs $K_{1}$ and $K_{2}$; $P_{2}$ : the vibrator contacts with spring $K_{1}$ but not with spring $K_{2} ; P_{3}$ : the vibrator contacts with spring $K_{2} ; P_{4}$ : the vibrator changes direction and returns after contacting with spring $K_{1} ; P_{5}$ : the vibrator is detached from spring $K_{2}$. Let $D P_{1}$, $D P_{2}, D P_{3}, D P_{4}$, and $D P_{5}$ denote the mapping matrices of $P_{1}, P_{2}, P_{3}, P_{4}$, and $P_{5}$; among them,

$$
\begin{aligned}
D P_{k} & =\left[\frac{\partial f_{k i}}{\partial Y_{j}}\right]_{\left(\dot{x}_{k-1}, \tau_{k-1}\right)} ; i, j=1,2, k=1,2,3, \\
D P_{k} & =\left[\frac{\partial f_{k i}}{\partial Y_{j}}\right]_{\left(\dot{x}_{m}, \tau_{m}\right)} ; i, j=1,2, k=4, m=1(\text { or } k=5, m=3) .
\end{aligned}
$$

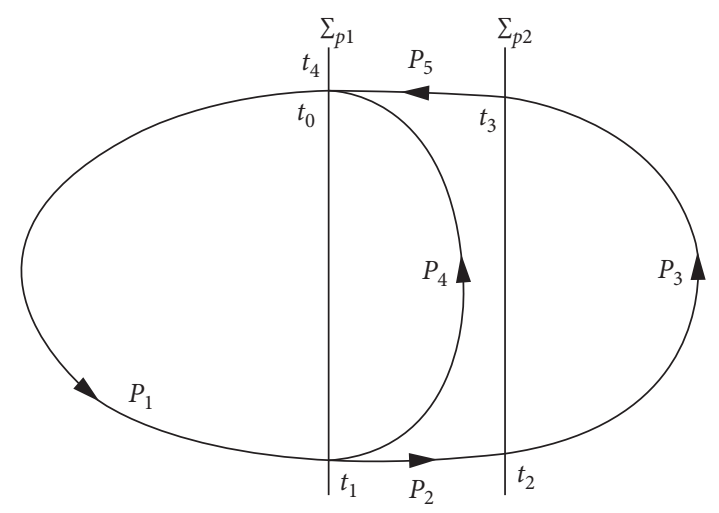

FIgURe 2: Schematic diagram of Poincaré mapping of the system.

In the above formulas, $f_{i}$ represents the $i$ component of $P_{k}$ and $Y_{j}$ represents the $j$ component of the coordinate. First, the integral constants $a_{l}$ and $b_{l}(l=1,2,3)$ are obtained from the initial conditions of the initial stages of each movement, and then, according to the termination conditions, $x\left(t_{1}\right)-b_{1}=0, x\left(t_{2}\right)-b_{1}-b_{2}=0, x\left(t_{3}\right)-b_{1}-b_{2}=0$, and $x\left(t_{4}\right)-b_{1}=0$. Then the Poincaré mapping of the system's $n-p-q$ period movement is

$$
\begin{aligned}
& \left\{\begin{array}{l}
f_{k 1}\left(\dot{x}_{k-1}, \tau_{k-1}\right)=\dot{x}(t), \\
f_{k 2}\left(\dot{x}_{k-1}, \tau_{k-1}\right)=\omega t+\tau_{k-1},
\end{array} \quad k \geq 3,\right. \\
& \left\{\begin{array}{l}
f_{k 1}\left(\dot{x}_{m}, \tau_{m}\right)=\dot{x}(t), \\
f_{k 2}\left(\dot{x}_{m}, \tau_{m}\right)=\omega t+\tau_{m},
\end{array}\right.
\end{aligned}
$$

Define the function:

$$
\begin{aligned}
G_{k}\left(t_{k}, \dot{x}_{k-1}, \tau_{k-1}\right) & =x\left(t_{k}\right)-b_{1}, & & k \geq 3, \\
G_{k}\left(t_{k}, \dot{x}_{m}, \tau_{m}\right) & =x\left(t_{k}\right)-b_{1}, & & k=4, m=1(\text { or } k=5, m=3) .
\end{aligned}
$$

According to the implicit function derivation theorem, each element in $D P_{k}$ can be found:

$$
a_{k i j}=\frac{\partial f_{k i}}{\partial Y_{j 0}}-\frac{\partial f_{k i}}{\partial t}\left(\frac{\partial G_{k}}{\partial Y_{j 0}} / \frac{\partial G_{k}}{\partial t}\right), \quad i, j=1,2 .
$$

The Lyapunov exponential spectrum is an effective tool for judging the stability and chaos of the dynamic system. In this paper, the Poincaré mapping method is used to transform the continuous dynamic system into a discrete dynamic system. The calculation formula is as follows:

$$
\lambda_{i}=\frac{1}{K} \sum_{k=1}^{K} \ln \left\|z_{i}^{(k)}\right\|, \quad i=1,2,
$$

where $K$ is the number of iterations, $z_{i}^{(k)}$ is the vector processed by Gram-Schmidt orthogonalization and norm normalization after each iteration, and $\left\|z_{i}^{(k)}\right\|(i=1,2)$ is the norm of $z_{i}^{(k)}$. 


\section{The Transition Law of Basic Periodic Motion}

In order to study the distribution type of the periodic motion of the system and its transition law, the gap $b_{1}$ and frequency $\omega$ are taken as the research objects. Take the system parameters $\zeta=0.1, \mu_{k 1}=20, \mu_{k 2}=50, \omega \in[0.1,2], b_{1} \in[0.1$, $2]$, and $b_{2}=0.1$. The periodic motion distribution of the system in the parameter plane $\left(\omega, b_{1}\right)$ is shown in Figure 3 and combined with the Lyapunov exponent to determine the existence of stable periodic motion and chaotic motion. In Figure 3, different colors and corresponding symbols are used to indicate the existence area and distribution law of various periodic motions. In symbol $n-p-q, n$ is the number of excitation cycles, $p$ is the number of times the vibrator contacts with the elastic constraint of right stiffness $K_{1}$ at forward speed, and $q$ is the number of times the vibrator contacts with the elastic constraint of right stiffness $K_{2}$ at forward speed. PF is used for period-doubling bifurcation, IPF for inverse period-doubling bifurcation, SN for saddle-node bifurcation, GR for edge rubbing bifurcation, and $\mathrm{BC}$ for the boundary crisis of the system. The areas not shown in the figure mainly include the following: (1) the parameter field which is too small and the display which is not obvious enough; (2) chaos area. In order to express the type of grazing bifurcation between the vibrator and different constraint surfaces, the following definitions are made:

(a) The grazing bifurcation between the vibrator and the elastic constraint surface with a stiffness coefficient $K_{1}$ is defined as the first type of grazing bifurcation, represented by GR1, and the corresponding grazing motion is defined as the first type of grazing periodic motion.

(b) The grazing bifurcation between the vibrator and the elastic constraint surface with a stiffness coefficient
$K_{2}$ is defined as the second type of grazing bifurcation, represented by GR2, and the corresponding grazing motion is defined as the second type of grazing periodic motion.

It can be seen from Figure 3(a) that, with the increase or decrease of $b_{1}$ and $\omega$, the basic periodic motion $1-p-q$ mainly has the following forms: $p$, the number of collisions between the vibrator and the elastic restraint surface with a stiffness coefficient $K_{1}$, is fixed, and $q$, the number of collisions with the elastic restraint surface with a stiffness coefficient $K_{2}$, gradually increases or decreases by one. The number of collisions $q$ between the vibrator and the elastic restraining surface with a stiffness coefficient $K_{2}$ is fixed; the number of collisions $p$ with the elastic restraint surface with a stiffness coefficient $K_{1}$ gradually increases or decreases by one. When the number of collisions gradually increases, the system will flutter. There are two main types of flutter: the first one is that flutters occur only between the oscillator and the elastic constrained surface with a stiffness coefficient $K_{1}$, which is expressed as $1-\tilde{p}-q$; the second type is that flutters occur between the oscillator and two kinds of elastic constrained surfaces, which is expressed as $1-\widetilde{p}-\widetilde{q}$. When both $p$ and $q$ are 0 , the vibrator does not collide with the two types of elastic constraints on the right side.

4.1. Analysis of the Basic Transition Law between Adjacent Periodic Motions. In the low-frequency small gap area, as shown in Figure 3(b), the $1-p-q$ periodic motion can be transferred to $1-(p+1)-q$ and $1-p-(q+1)$ periodic motion through the first and second types of grazing bifurcations, respectively.

Under the $b_{1}$ or $\omega$ change, the transition between $1-p-q$ and $1-(p+1)-q$ periodic motion conforms to the following rules:

$$
b_{1}(\omega) \downarrow: 1-(p+\infty)-q \stackrel{\mathrm{GR}_{1}}{\leftarrow} \cdots 1-(p+2)-q \stackrel{\mathrm{GR}_{1}}{\leftarrow} 1-(p+1)-q \stackrel{\mathrm{GR}_{1}}{\leftarrow} 1-p-q .
$$

Under the $b_{1}$ or $\omega$ change, the transition between $1-p-q$ and $1-p-(q+1)$ periodic motion conforms to the following rules:

$$
b_{1}(\omega) \downarrow: 1-p-(q+\infty) \stackrel{\mathrm{GR}_{2}}{\leftarrow} \cdots 1-p-(q+2) \stackrel{\mathrm{GR}_{2}}{\leftarrow} 1-p-(q+1) \stackrel{\mathrm{GR}_{2}}{\leftarrow} 1-p-q .
$$

Select $\omega=0.1722$ and $b_{1} \in[0.537,1.035]$ to analyze the transition process between $1-p-q$ and $1-(p+1)-q$ periodic motions in detail. It can be seen from Figure 4(a) that, in the process of large decrease of $b_{1}$, the first type of grazing bifurcation occurs in the system in turn, which causes the number of collisions $p$ between the vibrator and the elastic restraint surface with a stiffness coefficient $K_{1}$ to increase one by one. For example, the first type of grazing bifurcation occurs in the system at $b_{1}=0.80143$, and $1-4-0$ periodic motion transitions to $1-5-0$ periodic motion. Figure $5(\mathrm{a})$ is the phase diagram of the grazing periodic motion, and Figure $5(\mathrm{~b})$ is a partially enlarged view of the collision surface.

Select $\omega=0.2822$ and $b_{1} \in[0.1,0.2232]$ to specifically analyze the transition process between the $1-p-q$ and $1-p-(q+1)$ periodic motions. It can be seen from Figure $4(\mathrm{~b})$ that when $b_{1}$ reduces, the second type of grazing 


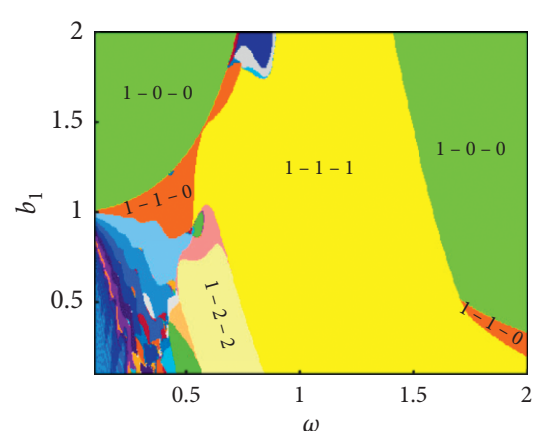

(a)

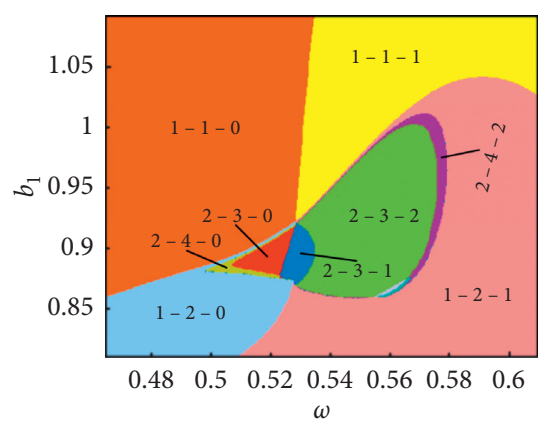

(d)

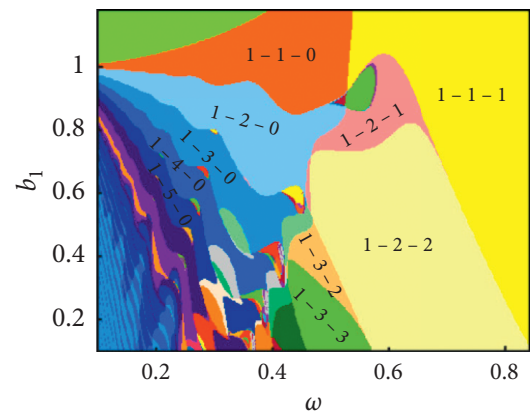

(b)

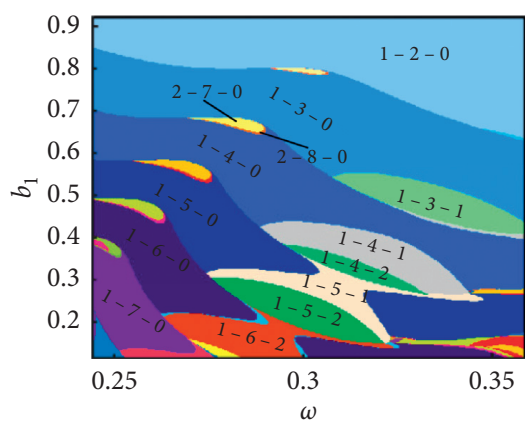

(e)

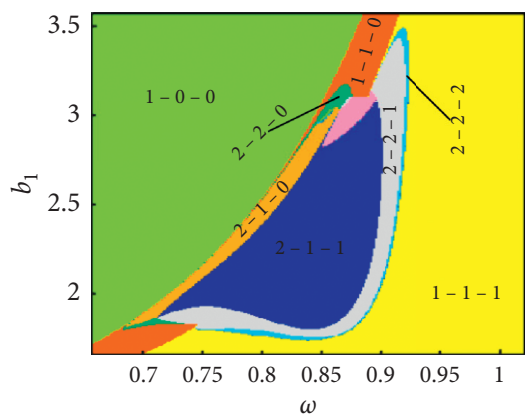

(c)

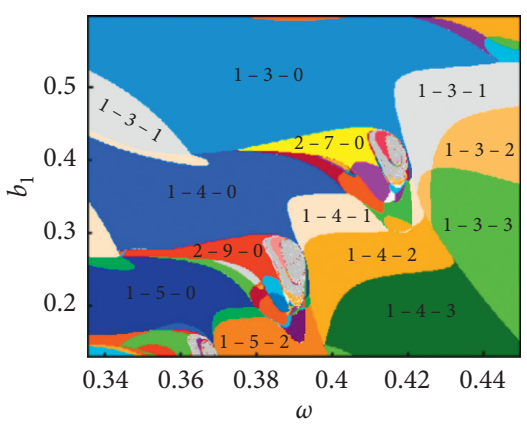

(f)

Figure 3: Distribution diagram of the periodic motion of the system in the parameter plane $\left(\omega, b_{1}\right)$. (a) Overall view. (b) Partial enlarged views 1. (c) Transitional zone between $1-0-0$ and $1-1-1$ periodic motions. (d) Partial enlarged views 2 of Figure 3(a). (e) Partial enlarged views 3 of Figure 3(a). (f) Partial enlarged views 4 of Figure 3(a).

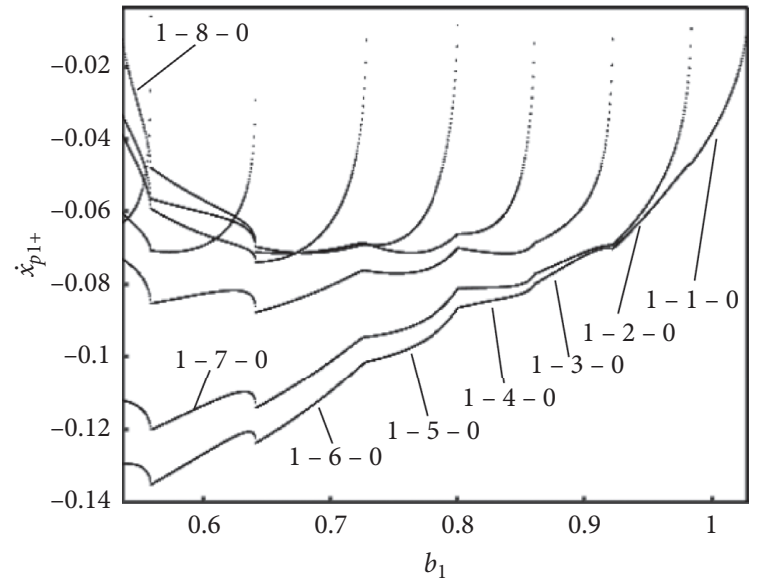

(a)

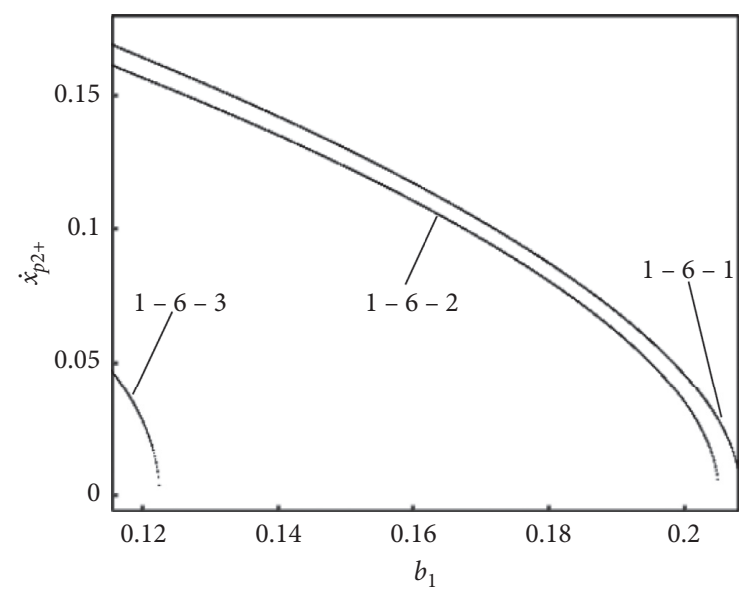

(b)

FIgURE 4: Single-parameter bifurcation diagram of the system. (a) $\omega=0.1722$. (b) $\omega=0.2822$.

bifurcation occurs in the system in turn, which causes the number of collisions $q$ between the vibrator and the elastic restraint surface with a stiffness coefficient $K_{2}$ to increase one by one. For example, the first type of grazing bifurcation occurs in the system at $b_{1}=0.12254$, and $1-6-2$ periodic motion transits to $1-6-3$ periodic motion. Figure $5(\mathrm{c})$ is a phase diagram of the periodic motion of the grazing, and Figure 5(d) is a partially enlarged view of the collision surface.
When adjacent periodic motions transfer to each other, because the parameter points of different types of bifurcation are not in the same position, the transfer process is irreversible, so that a polymorphic coexistence region composed of two adjacent periodic motions will be formed in the process of the transition of the two adjacent periodic motions. Take the system parameters $b_{1}=0.5826$ and $\omega=[0.6928,0.74]$ to analyze the polymorphic 


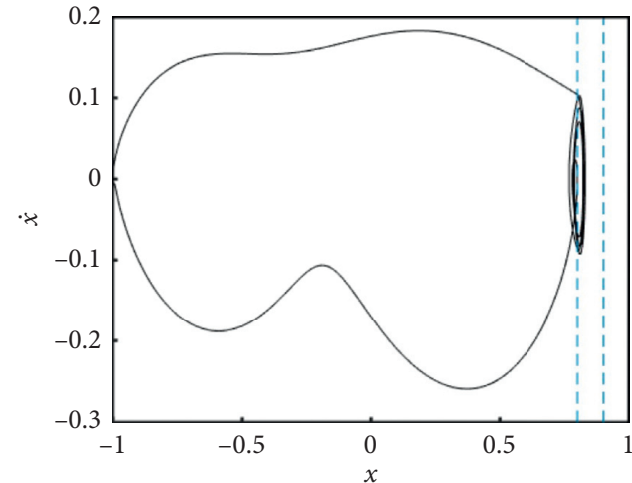

(a)

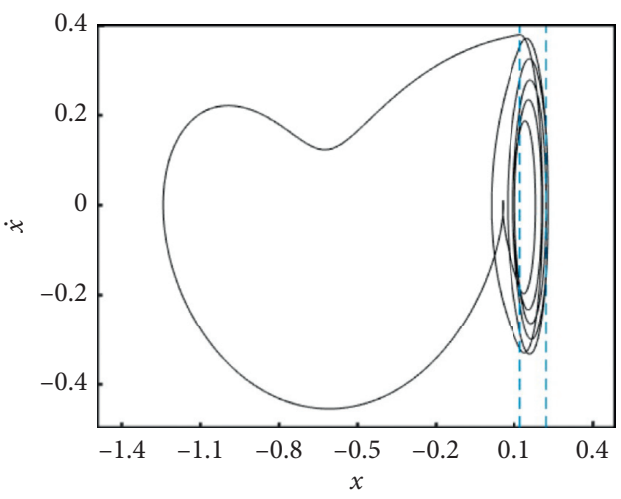

(c)

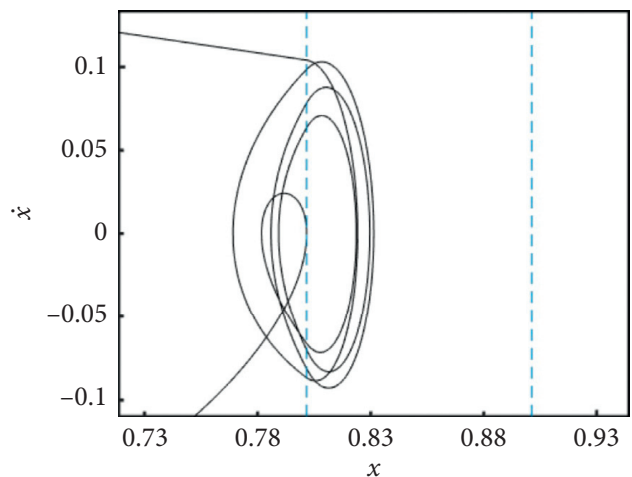

(b)

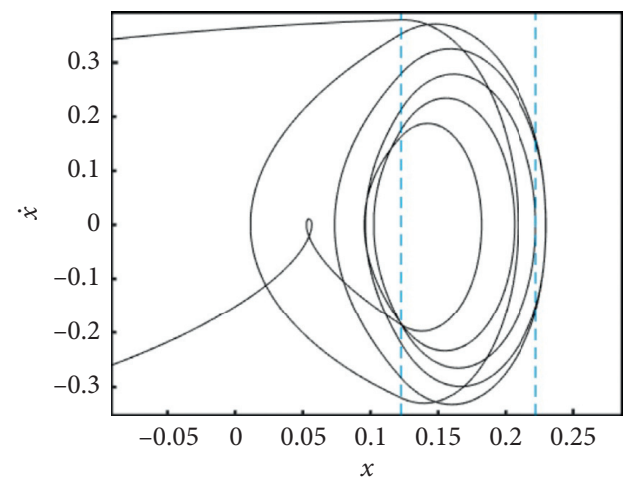

(d)

FIgURE 5: Phase diagram of periodic motion. (a) 1-4-0 phase diagram of grazing periodic motion. (b) Partially enlarged view of Figure 5(a). (c) 1-6-2 phase diagram of grazing periodic motion. (d) Partially enlarged view of Figure 5(d).

coexistence region when the $1-1-1$ and $1-2-2$ periodic motions transit mutually. Figure 6 is the bidirectional bifurcation diagram of the $\omega$ change, and the red curve shows the single-parameter bifurcation when $\omega$ is greatly reduced, and the black curve represents the single-parameter bifurcation diagram when $\omega$ increases gradually; it is convenient to observe the coexistence of different attractors. When $\omega$ decreases, the system transits from $1-$ $1-1$ periodic motion at $\omega=0.7092$ to $1-2-2$ periodic motion via the first type of grazing bifurcation; when $\omega$ increases, the system transits from $1-2-2$ periodic motion to $1-1-1$ via saddle-node bifurcation to $1-2-$ 2 cycles of movement. In this way, an area where $1-1-1$ and $1-2-2$ periodic motions coexist stably is formed between the two bifurcation points. Combined with the cell mapping method, select the initial state domain: $\Omega=\{(x, \dot{x}) \mid-2<x<-0.5,0<\dot{x}<2\}$, divide the initial state domain into $1000 \times 1000$ state cells, and the distribution diagram of the attractor of $\omega=0.715$ is shown in Figure 7.

4.2. Analysis of the Transition Law with Transition Zone between Adjacent Periodic Motions. In the parameter area shown in Figure 3(a), in addition to the above-mentioned basic transition methods between adjacent periodic motions, the transition process between adjacent basic periodic

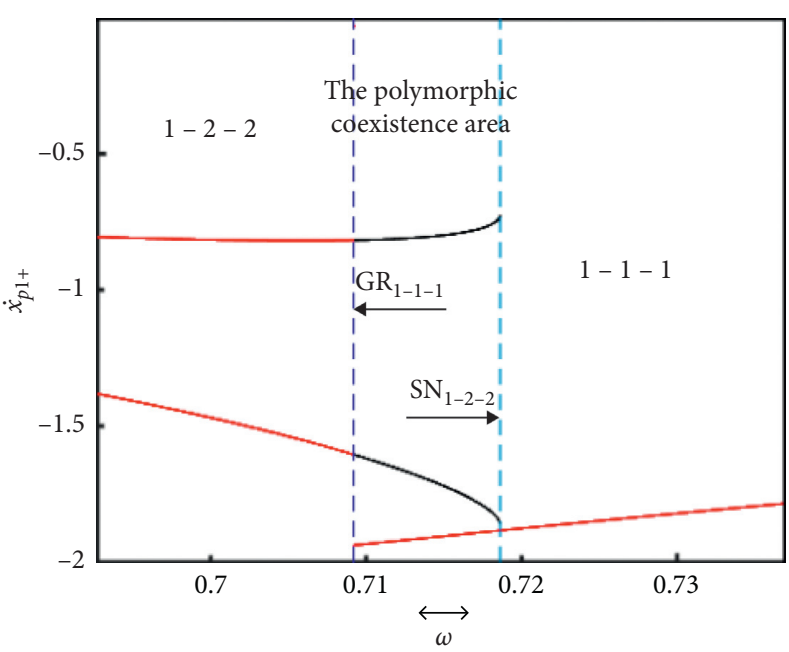

FIGURE 6: Bidirectional single-parameter bifurcation diagram of $b_{1}=0.5826$.

motions will be more complicated due to the emergence of the transition zone.

The first type of transition zone shown in Figure 3(e) generally exists at the peak position of the boundary line of two adjacent periodic motions. This type of transition zone has self-similar characteristics. The transition process of the 


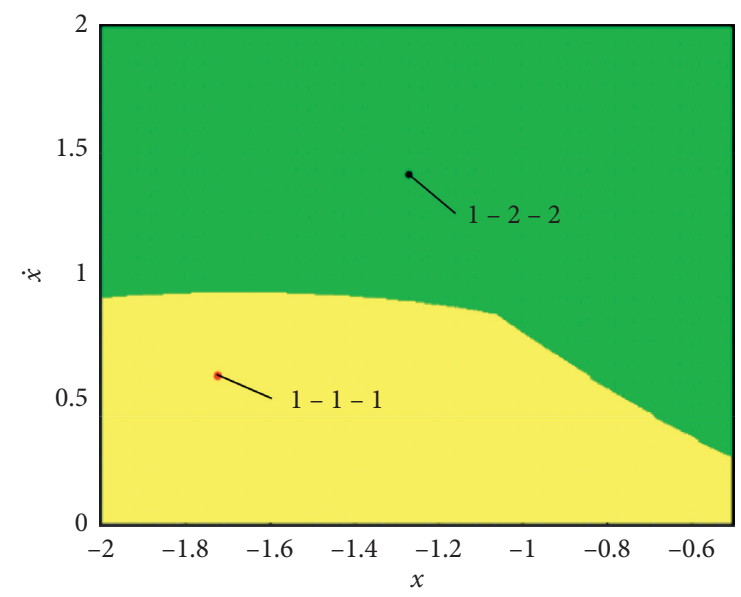

Figure 7: The attraction domain on the phase-fixing surface $\Sigma_{n}$ at $\omega=0.715$.

first type of transition zone is as follows: with the decrease of $b_{1}$, the system will have two grazing bifurcations of the first type in sequence, resulting in the basic periodic motion $1-p-q$ transiting to $2-(2 p+2)-2 q$ periodic motion and then to $1-(p+1)-q$ periodic motion via the inverse period-doubling bifurcation. The transitional process can be expressed as

$$
b_{1} \downarrow: 1-(p+1)-q \leftarrow 2-(2 p+2)-2 q \stackrel{\mathrm{IPB}}{\leftarrow} 2-(2 p+1)-2 q \stackrel{\mathrm{GR}_{1}}{\leftarrow} 1-p-q .
$$

Select $\omega=0.2706$ and $b_{1} \in[0.5261,0.5889]$ to analyze the process of $1-p-q$ periodic motion transiting to $1-$ $(p+1)-q$ periodic motion via the first transition zone. It can be seen from Figure 8 that, in the process of $b_{1}$ decreasing from large, $1-4-0$ periodic motion transits to $2-9-0$ periodic motion through the first type of grazing bifurcation at $b_{1}=0.5782$; the $2-9-0$ periodic motion transits to the $2-10-0$ periodic motion through the first type of grazing bifurcation at $b_{1}=0.5467$. Figure 9 (a) is the phase diagram of the $2-9-0$ grazing periodic motion, and Figure 9(b) is the partially enlarged view of the collision surface. Afterwards, the $2-10-0$ periodic motion transits to the $1-5-0$ periodic motion through the reverse period-doubling bifurcation.

The second type of transition zone shown in Figure 3(f) mainly exists at the intersection of multiple adjacent periodic motions. Due to the appearance of period-doubling bifurcation, $1-(p+1)-q$ and $1-p-(q+1)$ periodic motions will transit to the $2^{i}-2^{i}(p+1)-2^{i} q$ and $2^{i}-2^{i} p-2^{i}(q+1)$ periodic motions, respectively; $1-p-q$ will also transit directly to the chaotic motion via the saddle-node bifurcation. In this way, a transition zone with similar periodic motion distribution will be formed at the intersection of multiple adjacent periodic motions.

The parameters $b_{1}=0.2501$ and $w \in[0.382,0.396]$ are selected to specifically analyze the transitional process between various periodic motions in the transition zone. The single- parameter bifurcation diagram of the system and the Lyapunov exponent spectrum of the system are shown in Figures 10 and 11 . When $\omega$ decreases gradually, the system transits from $1-4-2$ periodic motion to chaos through saddle-node bifurcation and then transits to stable periodic motion through a series of inverse period-doubling bifurcations and then to $2-9-0$ periodic motion in the same way. After two first-type grazing bifurcations and two inverse period-doubling bifurcations, the system eventually transits to $1-5-0$ periodic motion. When $\omega$ increases gradually, the system transits from $1-5-0$ periodic motion to $2-9-0$ periodic motion through period-doubling bifurcation and saddle-node bifurcation and then to stable periodic motion through a series of period-doubling bifurcations and boundary crises and then enters chaos state, followed by transiting to $1-4-2$ periodic motion via a series of inverse perioddoubling bifurcations. It can be seen from Figure 11 that when $\omega$ increases gradually, the system enters the chaos state via saddle-node bifurcation at $\omega=0.3924$. The maximum Lyapunov exponent of the system is shown with blue line; at this point it changes from a negative number to a positive number; when $\omega$ decreases gradually, the system transits from chaos motion to $1-4-2$ periodic motion at $\omega=0.3935$ via a series of inverse period-doubling bifurcations; the maximum Lyapunov exponent of the system is shown with red line. After a series of swings at the zero-scale mark, the system stabilizes to a negative number, and the system is stable. The specific 


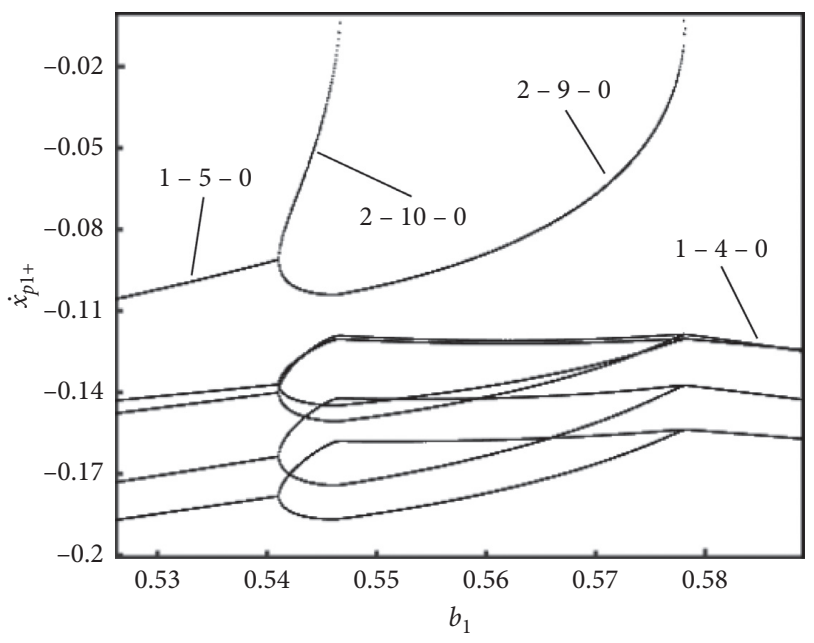

FiguRE 8: Single-parameter bifurcation diagram at $\omega=0.2706$.

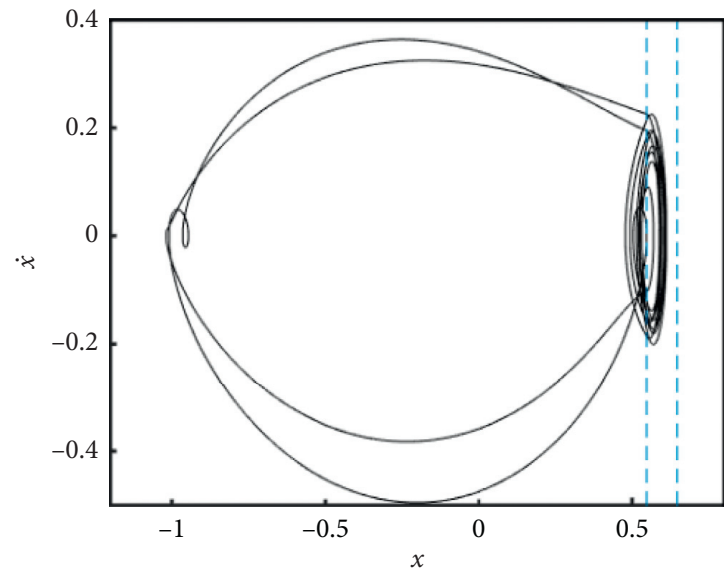

(a)

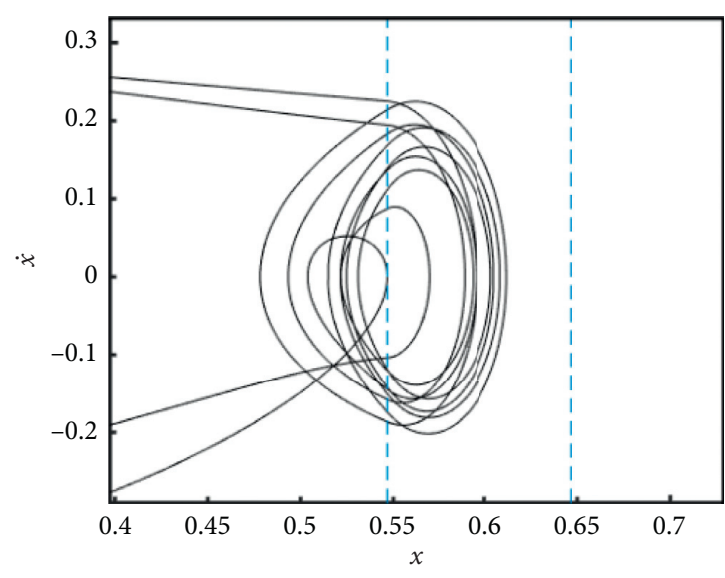

(b)

FIGURE 9: Phase diagram of the periodic motion. (a) 2-9-0 phase diagram of grazing periodic motion. (b) Partially enlarged view of Figure 9(a).

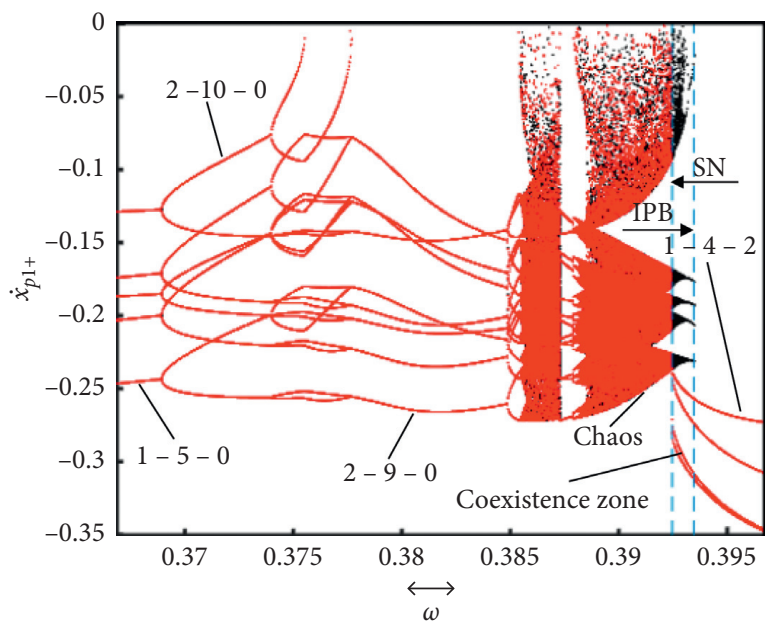

FIGURE 10: Bidirectional single parameter.

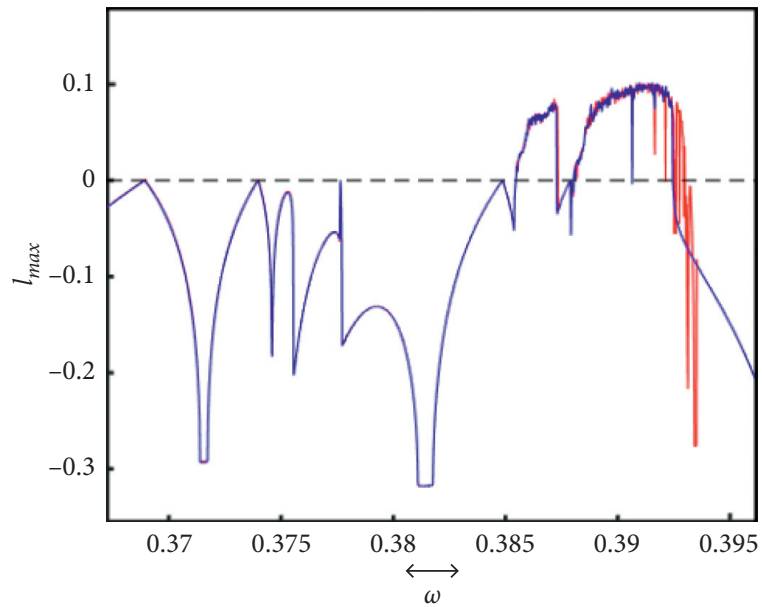

Figure 11: Maximum Lyapunov exponent of $b_{1}=0.2501$. 


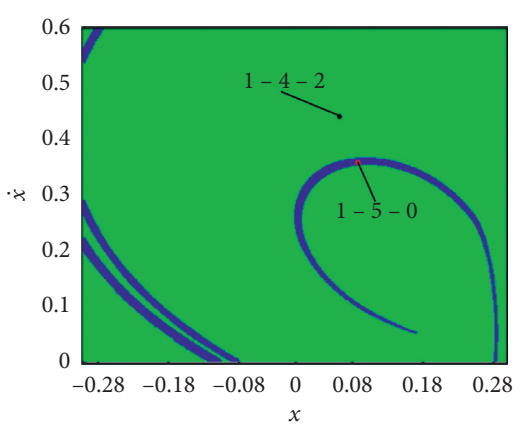

(a)

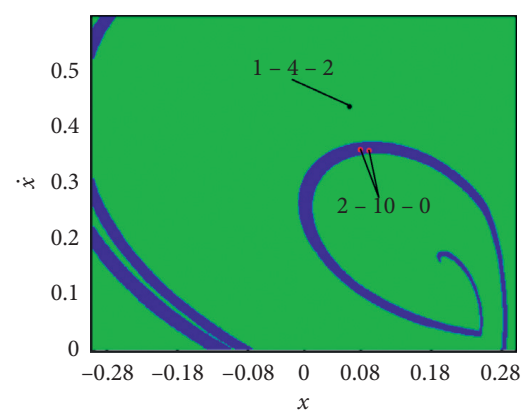

(b)

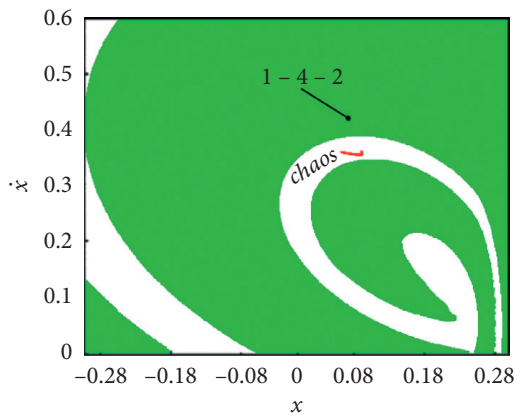

(c)

FIGURE 12: The attraction domain on the phase-fixed surface $\Sigma_{n}$. (a) $\omega=0.39347$. (b) $\omega=0.39357$. (c) $\omega=0.39259$

transitional process during the two-way change of $\omega$ is as follows:

$$
\begin{aligned}
& \omega \downarrow: 1-5-0 \stackrel{\mathrm{IPB}}{\longleftarrow} 2-10-0 \stackrel{\mathrm{IPB}}{\longleftarrow} 4-20-0 \stackrel{\mathrm{GR}_{1}}{\longleftarrow} 4-19-0 \stackrel{\mathrm{GR}_{1}}{\longleftarrow} 2-9-0 \stackrel{\mathrm{IPB}}{\longleftarrow} \cdots \stackrel{\mathrm{IPB}}{\longleftarrow} \text { chaos } \stackrel{\mathrm{SN}}{\longleftarrow} 3-13 \longleftarrow 0 \stackrel{\mathrm{IPB}}{\longleftarrow} \ldots \stackrel{\mathrm{IPB}}{\longleftarrow} \text { chaos } \stackrel{\mathrm{SN}}{\longleftarrow} 1-4-0, \\
& \omega \uparrow: 1-5-0 \stackrel{\mathrm{PB}}{\longrightarrow} 2-10-0 \longleftrightarrow \mathrm{PB}^{\mathrm{P}} 4-20-0 \stackrel{\mathrm{SN}}{\longrightarrow} 4-19-0 \stackrel{\mathrm{SN}}{\longrightarrow} 2-9-0 \stackrel{\mathrm{PB}}{\longrightarrow} \cdots \stackrel{\mathrm{PB}}{\longrightarrow} \text { chaos } \stackrel{\mathrm{BC}}{\longrightarrow} 3-13-0 \stackrel{\mathrm{PB}}{\longrightarrow} \cdots \stackrel{\mathrm{PB}}{\longrightarrow} \text { chaos } \stackrel{\mathrm{IPB}}{\longrightarrow} \cdots \stackrel{\mathrm{IPB}}{\longrightarrow} 1-4-2 .
\end{aligned}
$$

It can be seen from Figure 8 that since the saddle-node bifurcation and the inverse period-doubling bifurcation are not in the same position, there will be a polymorphic coexistence zone where the $1-4-2$ periodic motion and the chaotic motion coexist between the two parameter points. Combined with the cell mapping method, select the initial state domain: $\Omega=\{(x, \dot{x}) \mid-0.3<x<0.3,0<\dot{x}<0.6\}$, divide the initial state domain into $1000 \times 1000$ state cells, and the system periodic attractor distribution diagram is shown in Figure 12.

\section{The Influence of System Parameters on Periodic Motion Distribution}

This section uses the multiparameter collaborative simulation method to focus on the impact of the vibration system parameters, shown in Figure 1, on the periodic motion distribution and provides a theoretical basis for the reasonable design of matching parameters. The dynamic characteristics of the dimensionless equation (1) are mainly determined by the six parameters of $\zeta, \mu_{k 1}, \mu_{k 2}, b_{1}, b_{2}$, and $\omega$. Take the system parameters $\zeta=0.1, \mu_{k 1}=20, \mu_{k 2}=50$, $\omega \in[0.1,2], b_{1} \in[0.1,2]$, and $b_{2}=0.1$ as benchmark parameters. The influence of a certain parameter on the distribution of periodic motion of the system is analyzed and studied, taking discrete values within a reasonable range.

5.1. The Influence of Clearance $b_{2}$ on the Dynamic Characteristics of the System. The reference parameter of the clearance $b_{2}$ is 0.1 . For discrete values within a reasonable range, take $b_{2}=0.5, b_{2}=1.0$, and $b_{2}=1.5$ to calculate the distribution of periodic motion on the parameter plane $\left(\omega, b_{1}\right)$ under different $b_{2}$ values. Figures 13(a)-13(c) correspond to periodic motion distribution diagram under different values of $b_{2}$. By comparison with the reference parameter in Figure 3(a), it can be seen that when the clearance $b_{2}$ gradually increases, the system periodic motion parameter domain and its distribution law remain roughly unchanged, and the transition mode in the transition zone is basically the same. Comparing Figures $13(\mathrm{a})-13$ (c) with Figure 3(a), when $b_{2}$ increases to 0.5 , the $1-1-1$ periodic motion distribution area is significantly reduced, and it shifts to a larger place of $b_{1}$, there is no impact between the vibrator and the elastic constraint surface with a stiffness coefficient $K_{2}$, and the $1-p-0(p \geq 1)$ periodic motion distribution area is slightly widened. When $b_{2}$ increases to 1 , the $1-1-1$ periodic motion distribution area basically disappears and only exists in a part of the area at $b_{1}=1.6$. When $b_{2}$ increases to 1.5 , the distribution area of the $1-1-1$ periodic motion disappears completely, and the vibrator will have no impact on the elastic restraint surface with a stiffness coefficient $K_{2}$. At this time, the system can be regarded as only a kind of elastic restraint with a stiffness coefficient $K_{1}$.

5.2. The Influence of Damping Coefficient $\zeta$ on the Dynamic Characteristics of the System. The datum parameter of the damping coefficient $\zeta$ is 0.1 . Take discrete values for $\zeta$ within a reasonable range. Take $\zeta=0.05, \zeta=0.3$, and $\zeta=0.5$, respectively, to calculate the distribution of periodic motion on the $\left(\omega, b_{1}\right)$ parameter. Figures $14(\mathrm{a})-14(\mathrm{c})$ correspond to the periodic motion distribution diagram under different $\zeta$ values. Compared with the reference parameter in Figure 3(a), the change of damping coefficient $\zeta$ has a greater impact on the periodic motion distribution area. As shown in Figure 14(a), the peak value of each periodic motion generally increases, and the $1-2-2$ periodic motion distribution area is expanded to a larger clearance interval and the reduction of the damping coefficient of $\zeta$ causes the impact vibration system to present a very complex and fractal distribution law in the low-frequency range. The 


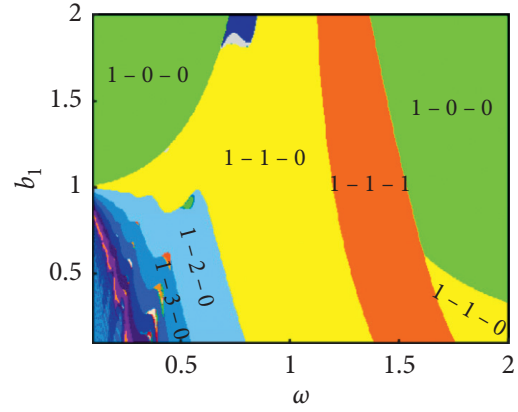

(a)

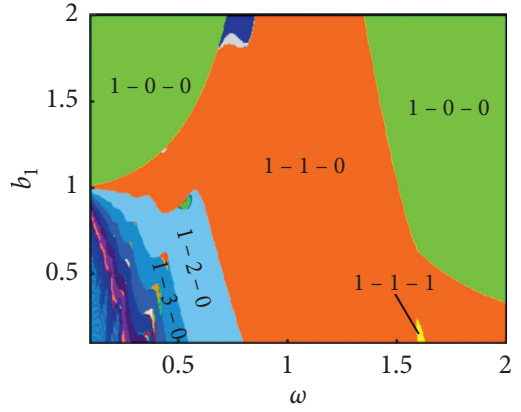

(b)

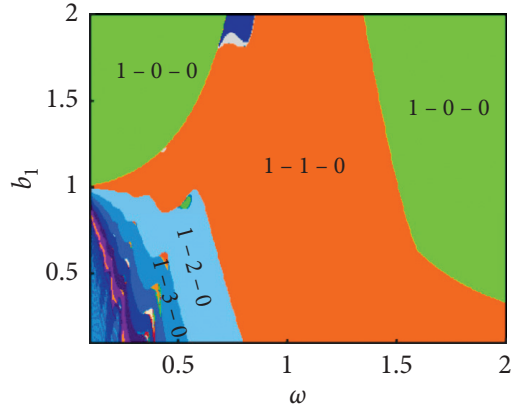

(c)

FIGURE 13: Distribution of periodic motion with $b_{2}$ change. (a) $b_{2}=0.5$. (b) $b_{2}=1.0$. (c) $b_{2}=1.5$.

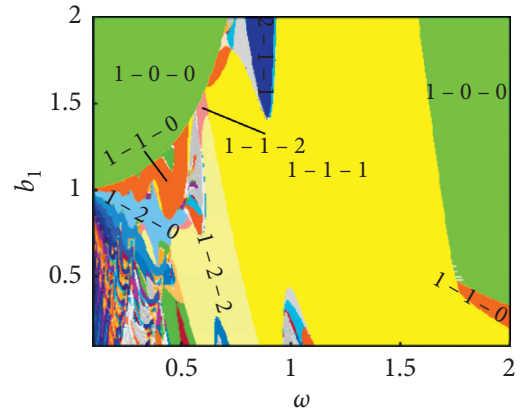

(a)

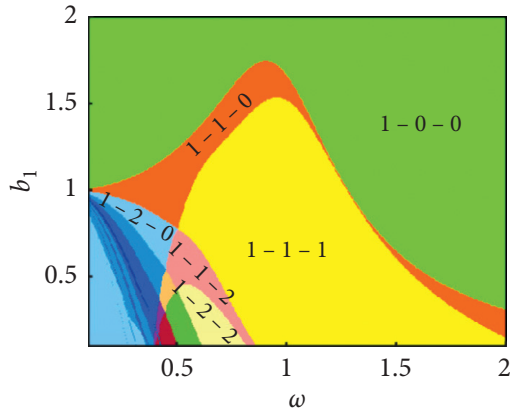

(b)

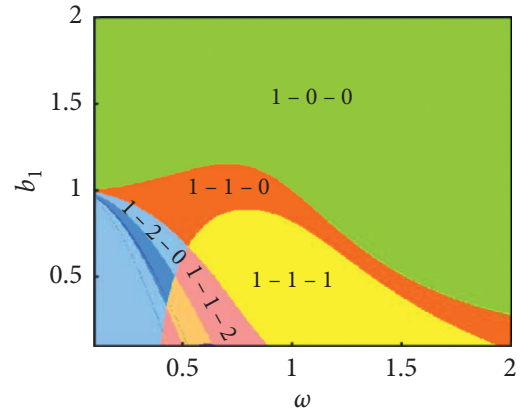

(c)

FIGURE 14: Distribution of periodic motion with $\zeta$ changes. (a) $k c=0.05$. (b) $k c=0.3$. (c) $k c=0.5$.

number of transition regions has increased significantly, the transition method is more complicated, and flutter and chaos occur in the system in many places. When the damping coefficient is $\zeta=0.3$, the dynamic characteristics of the system are no longer complicated. The transition between adjacent periodic motions is only through the basic transition method, the transition zone disappears, and the distribution area of $1-0-0$ periodic motion is slightly expanded. When the damping coefficient is $\zeta=0.5$, the types of basic periodic motion are further reduced, the peak value is generally reduced, and the distribution area of $1-0-0$ periodic motion is further expanded.

\section{Conclusions}

This paper takes a single-degree-of-freedom vibroimpact system with multilevel elastic constraints as the research object. By constructing the Poincare map, calculating the Lyapunov exponent spectrum to determine the mode type of the periodic motion of the system, the numerical simulation is carried out under the change of the system parameter $\left(\omega, b_{1}\right)$, and, combined with the cell mapping method, the law of transition between adjacent periodic motions of the system is analyzed. The influence of system parameters on the periodic motion distribution is analyzed.

(1) When directly transferring between adjacent periodic motions, in the process of $b_{1}$ and $\omega$ decreasing, $1-$ $p-q$ periodic motion can be transferred to $1-(p+$ 1) $-q$ and $1-p-(q+1)$ periodic motions through the first and second types of grazing bifurcations, respectively. When $b_{1}$ and $\omega$ increase, $1-(p+1)-q$ and $1-p-(q+1)$ periodic motions can be transferred to $1-p-q$ periodic motion through saddlenode bifurcation. When the adjacent periodic motion transits through the transition zone, the $1-p-q$ periodic motion transits to $2^{i}-2^{i} p-2^{i} q$ via perioddoubling bifurcation, to $2-2 p-(2 q+1)$ via the grazing bifurcation, or to the chaotic motion via the saddle-node bifurcation. Due to the different positions of different types of bifurcations in the system, the transition process is irreversible. In this way, a polymorphic coexistence zone, composed of two adjacent periodic motions, will be formed during the transition of adjacent periodic motions.

(2) By selecting different parameters and comparing them with benchmark parameters, the influence of each parameter on the distribution of system periodic motion is analyzed. The change of gap $b_{2}$ will change the periodic motion type of the system. With the increase of $b_{2}$, the area where the vibrator collides with the elastic restraint surface with a stiffness coefficient $K_{2}$ gradually decreases. When the collision area is $b_{2}=2$, the collision area completely disappears. It is equivalent to the fact that there is 
only one elastic constraint surface with stiffness coefficient $K_{1}$ in the system. The change of the damping coefficient $\zeta$ will have a greater impact on the periodic motion distribution area, and a smaller damping coefficient makes the basic periodic motion extremely complex and fractal at low frequencies, larger damping will cause the system to have only basic periodic motion, and there is no transition zone transition between adjacent periodic motions.

A future study in this field may include chaos control, bifurcation control, and coexistence attractor transition control of the systems with multiple parameters and multiple constraints. The study on the global dynamic characteristics of high-dimensional vibroimpact system is of great importance to the solution of practical engineering problems.

\section{Data Availability}

All the data of this research are reflected in the paper. Readers who are interested in this research can contact the leading author via e-mail: 37194175@qq.com.

\section{Conflicts of Interest}

The authors declare that there are no conflicts of interest regarding the publication of this paper.

\section{Acknowledgments}

This work was supported by the National Natural Science Foundation of China (nos. 11962013 and 11462011) and the Young Scholars Science Foundation of Lanzhou Jiaotong University (no. 2013019).

\section{References}

[1] W. Ding and J. Xie, "Torus and its routes to chaos of a vibroimpact system," Physics Letters A, vol. 349, no. 5, pp. 324-330, 2006.

[2] H. L. Yao, Y. B. Cao, S. J. Zhang, and B. C. Wen, "A novel energy sink with piecewise linear stiffness," Nonlinear Dynamics, vol. 94, no. 94, pp. 2265-2275, 2018.

[3] A. Fsharfard and A. Farshidianfar, "Design of nonlinear impact dampers based on acoustic and damping behavior," International Journal of Mechanical Sciences, vol. 65, no. 1, pp. 125-133, 2012.

[4] T. Zhang and H. Dai, "On the nonlinear dynamics of a highspeed railway vehicle with nonsmooth elements," Applied Mathematical Modelling, vol. 76, pp. 526-544, 2019.

[5] B. Guo, E. Ley, J. Tian, J. Zhang, Y. Liu, and S. Prasad, "Experimental and numerical studies of intestinal frictions for propulsive force optimisation of a vibro-impact capsule system," Nonlinear Dynamics, vol. 101, no. 1, pp. 65-83, 2020.

[6] S. W. Shaw and P. J. Holmes, "A periodically forced piecewise linear oscillator," Journal of Sound and Vibration, vol. 90, no. 1, pp. 129-155, 1983.

[7] S. Natsiavas, "Periodic response and stability of oscillators with symmetric trilinear restoring force," Journal of Sound and Vibration, vol. 134, no. 2, pp. 315-331, 1989.
[8] S. Natsiavas, "Steady state oscillations and stability of nonlinear dynamic vibration absorbers," Journal of Sound and Vibration, vol. 156, no. 2, pp. 227-245, 1992.

[9] Y. S. Choi and S. T. Noah, "Forced periodic vibration of unsymmetric piecewise-linear systems," Journal of Sound and Vibration, vol. 121, no. 1, pp. 117-126, 1988.

[10] Y. Ketema, "An oscillator with cubic and piecewise-linear springs," International Journal of Bifurcation and Chaos, vol. 1, no. 2, pp. 349-356, 1991.

[11] H. Y. Hu, "Numerical scheme of locating the periodic response of non-smooth non-autonomous systems of high dimension," Computer Methods in Applied Mechanics and Engineering, vol. 123, no. 1-4, pp. 53-62, 1995.

[12] A. C. J. Luo, "The mapping dynamics of periodic motions for a three-piecewise linear system under a periodic excitation," Journal of Sound and Vibration, vol. 283, no. 3-5, pp. 723748, 2005.

[13] A. B. Nordmark, "Non-periodic motion caused by grazing incidence in an impact oscillator," Journal of Sound and Vibration, vol. 145, no. 2, pp. 279-297, 1991.

[14] W. Chin, E. Ott, H. E. Nusse et al., "Universal behavior of impact oscillators near grazing incidence," Physics Letters A, vol. 201, no. 2-3, pp. 197-204, 1995.

[15] D. R. J. Chillingworth, "Dynamics of an impact oscillator near a degenerate graze," Nonlinearity, vol. 23, no. 11, pp. 2723-2748, 2010.

[16] N. Humphries and P. T. Piiroinen, "A discontinuity-geometry view of the relationship between saddle-node and grazing bifurcations," Physica D: Nonlinear Phenomena, vol. 241, no. 22, pp. 1911-1918, 2012.

[17] S. Kryzhevich and M. Wiercigroch, "Topology of vibro-impact systems in the neighborhood of grazing," Physica D: Nonlinear Phenomena, vol. 241, no. 22, pp. 1919-1931, 2012.

[18] E. Pavlovskaia, J. Ing, M. Wiercigroch, and S. Banerjee, "Complex dynamics of bilinear oscillator close to grazing," International Journal of Bifurcation and Chaos, vol. 20, no. 11, pp. 3801-3817, 2010.

[19] H. Jiang, A. S. E. Chong, Y. Ueda, and M. Wiercigroch, "Grazing-induced bifurcations in impact oscillators with elastic and rigid constraints," International Journal of Mechanical Sciences, vol. 127, no. 2, pp. 204-214, 2017.

[20] C. S. Hsu and R. S. Guttalu, "An unravelling algorithm for global analysis of dynamical systems: an application of cell-tocell mappings," Journal of Applied Mechanics, vol. 47, no. 4, pp. 940-948, 1980.

[21] J. Levitas, T. Weller, and J. Singer, "Poincaré-like simple cell mapping for non-linear dynamical systems," Journal of Sound and Vibration, vol. 176, no. 5, pp. 641-662, 1994.

[22] B. H. Tongue and K. Gu, "Interpolated cell Mapping of dynamical systems," Journal of Applied Mechanics, vol. 55, no. 2, pp. 461-466, 1988.

[23] W. Xu, Q. He, and S. Li, "The cell mapping method for approximating the invariant manifolds," in Proceedings of the Iutam Symposium on Dynamics and Control of Nonlinear Systems with Uncertainty, pp. 117-126, Nanjing, China, September 2006.

[24] C. Kong, X. Gao, and X. B. Liu, "On the global analysis of a piecewise linear system that is excited by a Gaussian white noise," Journal of Computational and Nonlinear Dynamics, vol. 11, no. 5, Article ID 051029, 2016.

[25] G. Li and W. Ding, "Global behavior of a vibro-impact system with asymmetric clearances," Journal of Sound and Vibration, vol. 423, pp. 180-194, 2018. 
[26] G. Li, J. Sun, and W. Ding, "Dynamics of a vibro-impact system by the global analysis method in parameter-state space," Nonlinear Dynamics, vol. 97, no. 1, pp. 541-557, 2019.

[27] A. S. E. Chong, Y. Yue, E. Pavlovskaia, and M. Wiercigroch, "Global dynamics of a harmonically excited oscillator with a play: numerical studies," International Journal of Non-Linear Mechanics, vol. 94, no. 9, pp. 98-108, 2017. 